\title{
Li-Decorated $\beta_{12}$-Borophene as Potential Candidates for Hydrogen Storage: A First-Principle Study
}

\author{
Tingting Liu ${ }^{1,2}$, Yuhong Chen ${ }^{1,2, *}$, Haifeng Wang ${ }^{3}$, Meiling Zhang ${ }^{2,4}$, Lihua Yuan ${ }^{2}$ and \\ Cairong Zhang ${ }^{1,2}$ \\ 1 State Key Laboratory of Advanced Processing and Recycling of No-ferrous Metals, \\ Lanzhou University of Technology, Lanzhou 730050, China; ttlLUT@163.com (T.L.); zhcrxy@lut.cn (C.Z.) \\ 2 School of Science, Lanzhou University of Technology, Lanzhou 730050, China; \\ zhangml_2000@126.com (M.Z.); yuanlh@lut.cn (L.Y.) \\ 3 Department of Physics, College of Science, Shihezi University, Xinjiang 832003, China; whfeng@shzu.edu.cn \\ 4 The School of Nuclear Science and Technology, Lanzhou University, Lanzhou 730000, China \\ * Correspondence: lzchenyh@163.com; Tel.: +86-931-297-3780
}

Received: 15 November 2017; Accepted: 4 December 2017; Published: 7 December 2017

\begin{abstract}
The hydrogen storage properties of pristine $\beta_{12}$-borophene and Li-decorated $\beta_{12}$-borophene are systemically investigated by means of first-principles calculations based on density functional theory. The adsorption sites, adsorption energies, electronic structures, and hydrogen storage performance of pristine $\beta_{12}$-borophene $/ \mathrm{H}_{2}$ and $\mathrm{Li}-\beta_{12}$-borophene $/ \mathrm{H}_{2}$ systems are discussed in detail. The results show that $\mathrm{H}_{2}$ is dissociated into Two $\mathrm{H}$ atoms that are then chemisorbed on $\beta_{12}$-borophene via strong covalent bonds. Then, we use Li atom to improve the hydrogen storage performance and modify the hydrogen storage capacity of $\beta_{12}$-borophene. Our numerical calculation shows that $\mathrm{Li}-\beta_{12}$-borophene system can adsorb up to $7 \mathrm{H}_{2}$ molecules; while $2 \mathrm{Li}$ - $\beta_{12}$-borophene system can adsorb up to $14 \mathrm{H}_{2}$ molecules and the hydrogen storage capacity up to $10.85 \mathrm{wt} \%$.
\end{abstract}

Keywords: $\beta_{12}$-borophene; Li-decorated; hydrogen storage; first-principles calculations

\section{Introduction}

As the gap between energy supply and demand has become increasingly prominent, sources of renewable energy has been investigated urgently. Hydrogen is an inexhaustible source of clean energy, making it important for society to develop and utilize this energy [1,2]. Hydrogen storage is one of the most critical technical problems in the development of hydrogen energy sources. The average adsorption energy of the ideal physical hydrogen storage method should be between chemical and physical adsorption energy $(0.1 \sim 0.8 \mathrm{eV})[3,4]$. The US Department of Energy (DOE) and the International Energy Agency (IEA) reported that the ideal hydrogen storage capacity should be greater than $5.5 \mathrm{wt} \%$ [5]. At present, one of the best types of hydrogen storage methods involves physical adsorption, which results in low adsorption heat, small activation energy, fast hydrogen adsorption and desorption, and reversible cyclization performance. Carbon nanomaterials have become a hotspot of physical hydrogen storage materials due to their characteristics of a large specific surface area, good adsorption kinetic properties and reversible hydrogen storage [6,7]. However, clean carbon nanomaterials adsorb $\mathrm{H}_{2}$ molecules with weak binding capacity, which means that they have low hydrogen storage capacity and are not ideal. Therefore, it is essential to find a suitable physical adsorbent.

Recently, 2D (two-dimensional) borophene created from Boron elements was artificially synthesized [8]. Although there are many theoretical studies about the possible 2D borophene structure [9], only three types of stable structures have been synthesized for borophene so far $[8,10]$. Borophene's unique metal properties, mechanical properties, and optical properties have 
been extensively studied [11-15], but only a few studies have considered its hydrogen storage properties. Borophene and graphene [16] have a similar 2D planar structure with a large specific surface area. Moreover, the relative atomic mass of $\mathrm{B}$ atom is smaller than the relative atomic mass of $\mathrm{C}$ atom. Therefore, we suspect that borophene has better hydrogen storage properties than graphene (it exhibits a triangular lattice with different periodic arrangements and is flat without obvious vertical undulation). Feng et al. [10] reported that $\beta_{12}$-borophene is more stable than the other two types of borophene. Chen et al. [17] used the first-principles method to study the hydrogen storage properties of $\mathrm{Ca}-\beta_{12}$-borophene and found that it has a larger adsorption energy compared to other types of borophene. Therefore, we selected $\beta_{12}$-borophene as the research focus. In this work, we performed theoretical calculations for the hydrogen storage properties of pure $\beta_{12}$-borophene and $\mathrm{Li}-\beta_{12}$-borophene based on the first-principle study. We found that $\mathrm{H}_{2}$ molecules were completely dissociated into two $\mathrm{H}$ atoms that were adsorbed on the $\mathrm{B}-\mathrm{B}$ bridge sites to form $\mathrm{H}-\mathrm{B}$ covalent bonds, thus making it difficult to dissociate. Comparison of the improvement in hydrogen storage properties of graphene found that the graphene surface was modified by alkali metal ( $\mathrm{Li}, \mathrm{Na}, \mathrm{K})$ [18], alkali-earth metal (Ca) [19], light metal (Al) [20] and transition metals (Cu, Pd, Y) [21-24], which can change the chemical activity of the graphene surface and could effectively change the hydrogen storagecapability. The quality of alkali metal ( $\mathrm{Li}$ atoms) is very light, which helps to enhance the hydrogen storage density [25]. The transition metal atom-modified nanostructures are highly reactive and can easily cause the dissociation of $\mathrm{H}_{2}$ molecules, which is detrimental to the reversible storage of hydrogen [26]. Therefore, we selected the lightest Li atom to modify the $\beta_{12}$-borophenen. $\mathrm{H}_{2}$ adsorbed on Li- $\beta_{12}$-borophene by physical adsorption, which improved the reversible hydrogen storage performance and significantly increased the amount of hydrogen storage. It is expected that this work can provide theoretical support for $\beta_{12}$-borophene being used as hydrogen materials.

\section{Computational Methods}

All density functional theory (DFT) calculations are carried out using the Cambridge Sequential Total Energy Package (CASTEP) [27], and the DFT evaluation is based on the plane-wave expansion. We use the Generalized Gradient Approximation (GGA) with the Perdew-Burke-Ernzerhof (PBE) exchange-correlation functional [28] to describe exchange and correlation effects. The van der waals forces of $\mathrm{H}_{2}$ adsorption on $\mathrm{Li}-\beta_{12}$-borophenen is modified by DFT-D methods. While the DFT-D perform poorly for energetics in layered materials [29], it is important to deal with the molecules adsorption system. We select the Ultrasoft Pseudopotential [30] to describe the interaction of electron-ion, and the electron wave functions are expanded by plane wave. The convergence tolerance energy, the force on each atoms and displacement convergence criterions are set to $5.0 \times 10^{-6} \mathrm{eV} / \mathrm{atom}$, $0.01 \mathrm{eV} / \AA$ and $0.001 \AA$, respectively. All atoms are relaxed in our calculations. In order to eliminate the interaction of the interlayer we select the vacuum thickness $20 \AA$. Considering the calculation accuracy and computational efficiency, all calculations are using a cutoff energy of $600 \mathrm{eV}$ and $9 \times 16 \times 5$ k-point mesh in the Brillouin zone.

The adsorption energy $\left(E_{a d s}\right)$ and average adsorption energy $\left(\bar{E}_{a d s}\right)$ of $\mathrm{H}_{2}$ adsorption on $\mathrm{Li}-\beta_{12}$-borophene are calculated by the following formulas [31]:

$$
\begin{gathered}
E_{a d s}=E_{i \mathrm{H} 2+n L i+\beta 12-\text { borophene }}-E_{(i-1) \mathrm{H} 2+n \mathrm{Li}+\beta 12-\text { borophene }}-E_{\mathrm{H} 2} \\
\overline{E_{a d s}}=\left(E_{i \mathrm{H} 2+n \mathrm{Li}+\beta 12-\text { borophene }}-E_{n \mathrm{Li}+\beta 12 \text {-borophene }}-i E_{\mathrm{H} 2}\right) / i
\end{gathered}
$$

The average adsorption energy of $\mathrm{Li}$ atom on $\beta_{12}$-borophenec [32] is defined as:

$$
\overline{E_{b}}=\left(E_{n \mathrm{Li}+\beta 12-\text { borophene }}-E_{\beta 12-\text { borophene }}-n E_{\mathrm{Li}}\right) / n
$$

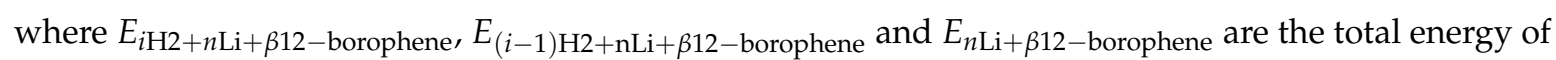
the $n \mathrm{Li}-\beta_{12}$-borophene with $i, i-1 \mathrm{H}_{2}$ molecules and $\beta_{12}$-borophene with $n \mathrm{Li}$ atoms, respectively. 
$E_{\beta 12 \text {-borophene, }} E_{\mathrm{Li}}$ and $E_{\mathrm{H} 2}$ are the total energy of the $\beta_{12}$-borophene, free $\mathrm{Li}$ atom and an isolated $\mathrm{H}_{2}$, respectively. $n$ is the number of adsorbed Li atoms.

\section{Results and Discussion}

\section{1. $H_{2}$ Adsorption on $\beta_{12}$-Borophene}

The optimized lattice parameters of the primitive cell of $\beta_{12}$-borophenen are a $=5.069 \AA$, $\mathrm{b}=2.929 \AA$, agree well with the experimental result ( $\mathrm{a}=5 \AA$ and $\mathrm{b}=3 \AA$ ) [10] and other theoretical calculation results [33-35]. In our follow calculations, we choose a $2 \times 2$ unit cell (see Figure 1 ) of the $\beta_{12}$-borophenen containing $20 \mathrm{~B}$ atoms (in Figure 1) to investigate the hydrogen storage adsorbed on $\beta_{12}$-borophene.

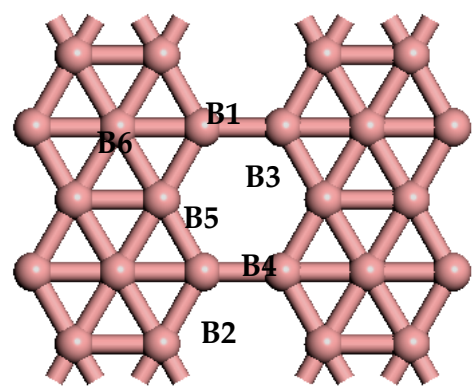

Figure 1. The optimized atomic structure of pure $\beta_{12}$-borophene. The alphanumeric characters on the graph represent the corresponding atoms.

We first investigated the adsorption behavior of one $\mathrm{H}_{2}$ molecule on $\beta_{12}$-borophene. The $\mathrm{H}_{2}$ molecule is initially placed in a parallel or vertical direction at different positions of the $\beta_{12}$-borophene plane. We found that there are five stable adsorbed configurations in total, as illustrated in Figure 2. In all cases, the $\mathrm{H}_{2}$ molecule is dissociated into two separate $\mathrm{H}$ atoms after adsorption and the distance between the $\mathrm{H}$ atoms will change from 0.753 to $2.366 \AA$. Furthermore, the distance between $\mathrm{H}$ and its nearest $\mathrm{B}$ atom $\left(\mathrm{r}_{\mathrm{H}-\mathrm{B}}\right)$ greatly increased from 1.217 to $1.358 \AA$. The most stable case among all the five adsorption configurations is shown in Figure 2a. In this case, the $\mathrm{H}_{2}$ molecule dissociated into two $\mathrm{H}$ atoms that are adsorbed on the B1-B3 and B2-B4 bridge sites with an $E_{a d s}$ value of $-0.536 \mathrm{eV}$, which is related to chemical adsorption. Mulliken analysis demonstrates that there is $0.23 \mathrm{e}^{-}$transferred from $\mathrm{B}$ to $\mathrm{H}$, which occurs mainly in the $\mathrm{H}$ 1s orbital and $\mathrm{B} 2 \mathrm{p}$ orbital. The B-H bond population is 0.4 , indicating it is a covalent bond, with difficult desorption of the $\beta_{12}$-borophene $/ \mathrm{H}_{2}$ system. In addition, we further studied the transition states of the stable adsorption configurations by combining linear synchronous and quadratic synchronous transits [21,36]. We found that the most stable adsorption configurations of the activation energy barrier from the reactant to transition state was $1.584 \mathrm{eV}$, which is smaller than the activation energy barrier of other adsorption methods, indicating it was difficult for the reaction of the $\mathrm{H}_{2}$ molecules adsorbed on the surface to take place.

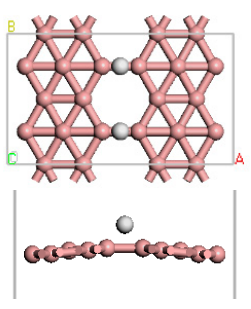

(a)

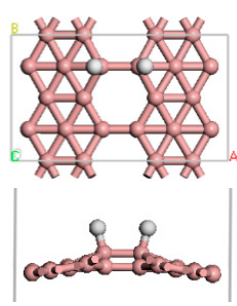

(b)

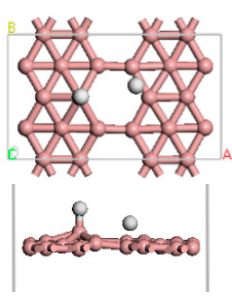

(c)

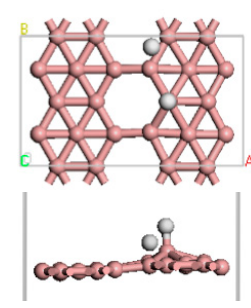

(d)

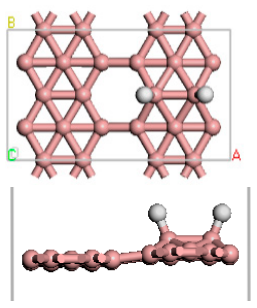

(e)

Figure 2. (a-e) show the five stable optimized geometrical structures of $\beta_{12}$-borophene $/ \mathrm{H}_{2}$. 


\section{2. $H_{2}$ Adsorption on Li- $\beta_{12}$-Borophene}

\subsubsection{The Adsorption Structure of Li- $\beta_{12}$-Borophene}

It is well known that doping alkali metal atoms to modify hydrogen storage materials may can greatly improve the hydrogen storage properties and increase the hydrogen storage capacity. Specially, lithium $(\mathrm{Li})$ has been widely employed to functionalize 2D materials and improve the hydrogen storage ability. Therefore, in the following section, we chose to add Li atoms to modify the hydrogen storage properties of $\beta_{12}$-borophene.

We examined the adsorption of Li atoms on pure $\beta_{12}$-borophene. After optimization, we obtained three different stable adsorption structures, as shown in Figure 3a-c. Similar to Li-decorating graphene [37], the most favorable $\mathrm{Li}$ adsorption site on $\beta_{12}$-borophene is the hollow center of $\mathrm{B}$ ring (Figure 3a).

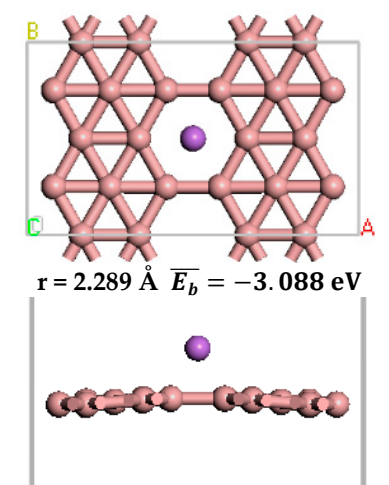

(a)

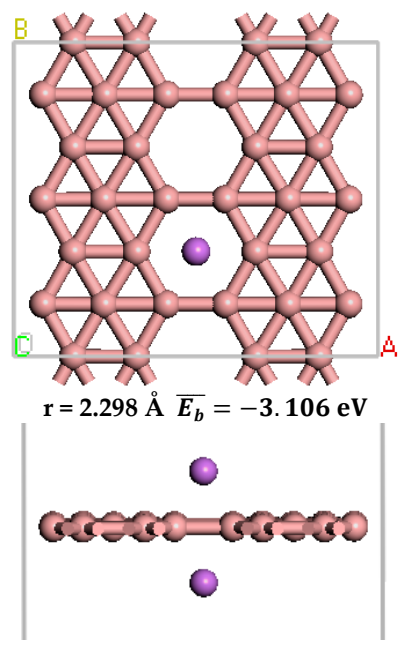

(d)

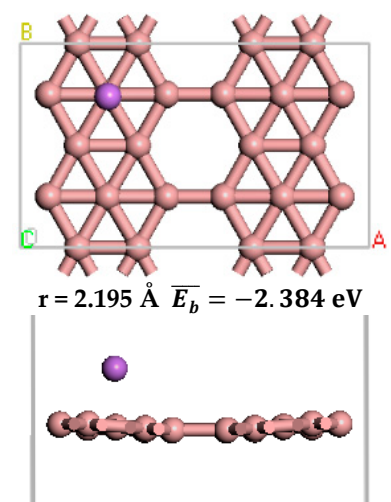

(b)

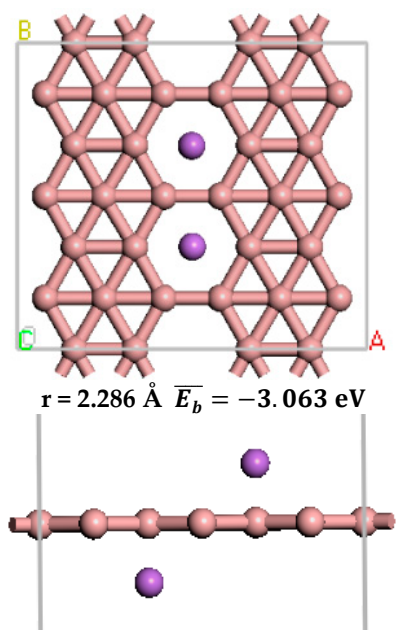

(e)

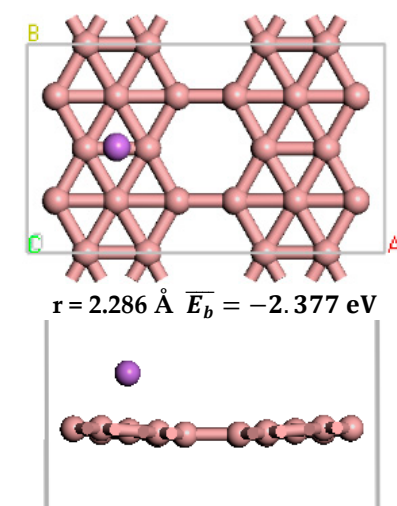

(c)

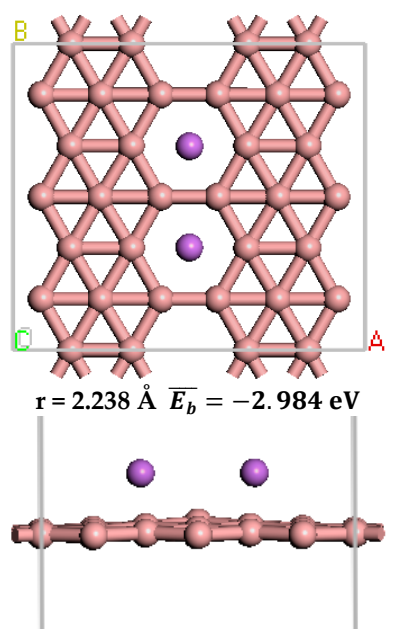

$(\mathbf{f})$

Figure 3. The optimized atomic structure of $\mathrm{Li}$ atom decorated $\beta_{12}$-borophene. (a-c) show the one Li atom decorated single-sided $\beta_{12}$-borophene, respectively. (d-f) show the two Li atoms decorated double-sided $\beta_{12}$-borophene, respectively.

Doping alkali metal atoms to modify hydrogen storage materials requires the average adsorption energy of the metal atoms on the substrate to be greater than the cohesive energy of the metal atoms in the solid form [38]. The average adsorption energy of Li atom on the $\beta_{12}$-borophene is $-3.088 \mathrm{eV}$, which is significantly greater than the cohesive energy of $-1.795 \mathrm{eV}$ of $\mathrm{Li}$ [39]. This indicates that $\mathrm{Li}$ atoms can be dispersed uniformly on $\beta_{12}$-borophene, instead of forming metal clusters. 
There are three stable adsorption structures of two Li atoms after adsorption on the $\beta_{12}$-borophene as shown in Figure $3 \mathrm{~d}-\mathrm{f}$, respectively. One of the most stable adsorption sites involves the two Li atoms being located on both sides of the same B ring. The distance between Li and the nearest B is $2.298 \AA$. The average adsorption energy is $-3.106 \mathrm{eV}$, which is larger than the cohesive energy of $\mathrm{Li}$ atoms. After optimization, the relaxation of $\beta_{12}$-borophene is very small. Each $\mathrm{Li}$ atom in the $\mathrm{Li}$ - $\beta_{12}$-borophene system is an active adsorption site, allowing a large number of $\mathrm{H}_{2}$ molecules to be adsorbed around the Li atom in order to significantly increase the hydrogen storage capacity.

The charge transfer between atoms can be analyzed by Mulliken analysis [40], which shows that the charge was transferred from Li to B. From the Partial Densities of States (PDOS) of the $\mathrm{Li}-\beta_{12}$-borophene structure in Figure 4 , we found the peak of B atom's $2 p$ orbital overlaps with the peak of the Li atom's 1s orbital. This suggests a strong hybridization between B and Li atoms. A similar binding mechanism has also been confirmed in other metal-modified nanostructures [41]. In addition, it can be seen from the PDOS that the metal properties of $\beta_{12}$-borophene did not change after modification of $\mathrm{Li}$ atom.

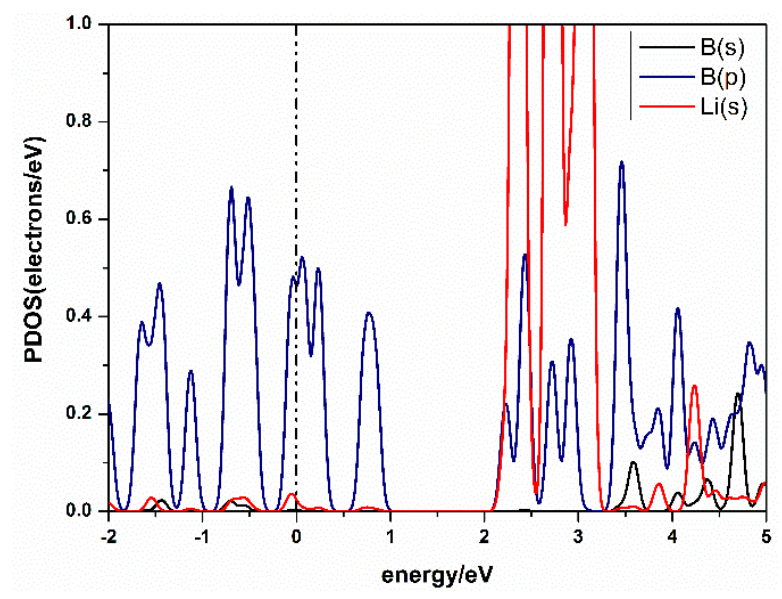

Figure 4. Partial density of states (PDOS) of Li-decorated $\beta_{12}$-borophene system.

\subsubsection{Adsorption of $\mathrm{H}_{2}$ Molecules on Li- $\beta_{12}$-Borophene}

We investigated the adsorption properties of $\mathrm{H}_{2}$ molecules on $\mathrm{Li}$ - $\beta_{12}$-borophene. Figure 5 shows the optimized geometries of 1-7 $\mathrm{H}_{2}$ molecules adsorbed on the Li-modified $\beta_{12}$-borophene. Table 1 lists the adsorption energy and average adsorption energy calculated by the GGA PBE functional and DFT-D methods. First, we investigate the adsorption sites of $\mathrm{H}_{2}$ molecules on $\mathrm{Li}-\beta_{12}$-borophene. For the first adsorbed $\mathrm{H}_{2}$ molecules, many adsorption sites were considered in order to find the most stable site. The most stable site involves $\mathrm{H}_{2}$ being parallel to the $\beta_{12}$-borophene plane, which is opposite to the $\mathrm{H}_{2}$ vertical adsorption on Ca- $\beta_{12}$-borophene [17]. After adsorption, the corresponding $\mathrm{r}_{\mathrm{H}-\mathrm{H}}$ of the adsorbed $\mathrm{H}_{2}$ is $0.756 \AA$, which is larger than the distance of free $\mathrm{H}_{2}(0.753 \AA)$. To investigate the maximum storage capacity of single Li atom-modified $\beta_{12}$-borophene, more $\mathrm{H}_{2}$ was added around Li gradually. The minimum distance between the $\mathrm{H}$ and $\mathrm{Li}$ atom are range of 2.164 to $6.368 \AA$. The first four $\mathrm{H}_{2}$ molecules were parallel to the $\beta_{12}$-borophene and were around the $\mathrm{Li}$ atom at the same level. When the fifth $\mathrm{H}_{2}$ molecule was added to the system, two $\mathrm{H}_{2}$ molecules moved to an upper layer after relaxation. This may be due to the limited space around the Li atom and the repulsive interactions between the adsorbed $\mathrm{H}_{2}$. The average adsorption energy slowly reduced from -0.385 to $-0.210 \mathrm{eV} / \mathrm{H}_{2}$ due to the strong steric interactions between the adsorbed $\mathrm{H}_{2}$. Interestingly, the adsorption energy suddenly rose to $-0.388 \mathrm{eV}$ after the second $\mathrm{H}_{2}$ molecule was added to the system. With an increase in the number of $\mathrm{H}_{2}$ molecules, the $\mathrm{H}_{2}$ molecules becomes further away from the $\mathrm{Li}$ atom and the adsorption weakens. The average adsorption energy was at its minimum $\left(-0.210 \mathrm{eV} / \mathrm{H}_{2}\right)$ when the seventh $\mathrm{H}_{2}$ molecule was adsorbed. At this time, the hydrogen storage capacity reached $5.90 \mathrm{wt} \%$, which exceeded the ideal 
hydrogen storage capacity (over $5.5 \mathrm{wt} \%$ ). In order to further increase the hydrogen storage capacity, we added two $\mathrm{Li}$ atoms to decorate the $\beta_{12}$-borophene to adsorb $\mathrm{H}_{2}$ molecules. $2 \mathrm{Li}$ - $\beta_{12}$-borophene can adsorb up to $14 \mathrm{H}_{2}$ molecules and the minimum average adsorption energy is $-0.220 \mathrm{eV}$. The hydrogen storage capacity can reach up to $10.85 \mathrm{wt} \%$, which is larger than the hydrogen storage capacity with a gravimetric hydrogen density of $9.5 \mathrm{wt} \%$ of the Ca- $\beta_{12}$-borophene $/ \mathrm{H}_{2}$ system [17]. The optimized structure is shown in Figure $5 \mathrm{~h}-\mathrm{n}$. The average adsorption energy $\left(\bar{E}_{\text {ads }}\right)$ is in the range of -0.381 to $-0.220 \mathrm{eV} / \mathrm{H}_{2}$, which is necessary for practical application [3,4]. In addition, the calculated adsorption energy and average energy of $\mathrm{H}_{2}$ adsorption by DFT-D method are larger than those calculated by the GGA PBE functional, but the overall adsorption method has not changed.

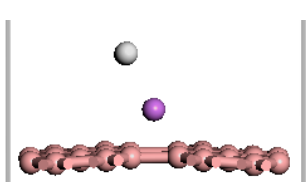

(a)

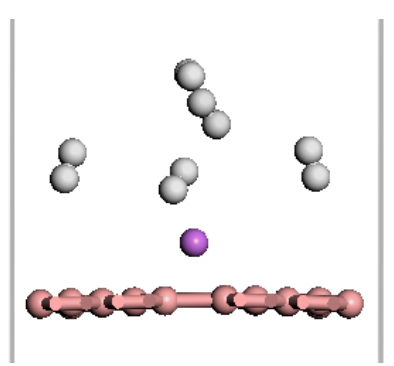

(e)

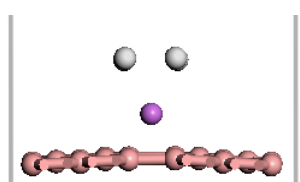

(b)

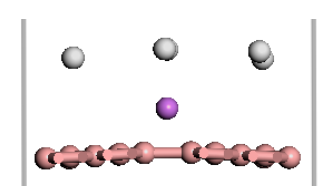

(c)

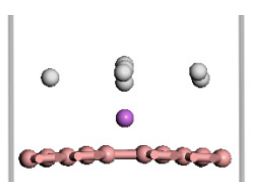

(d)

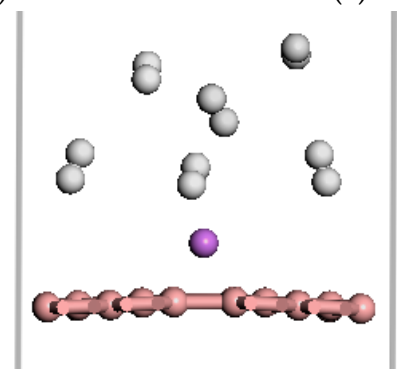

(f)

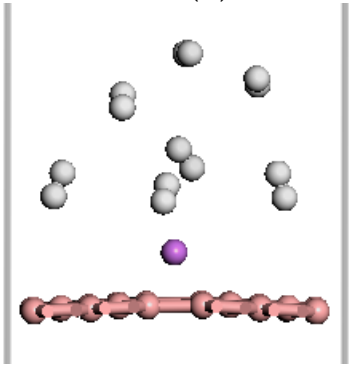

(g)

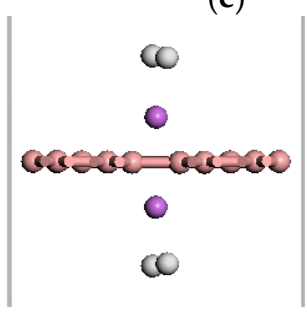

(h)

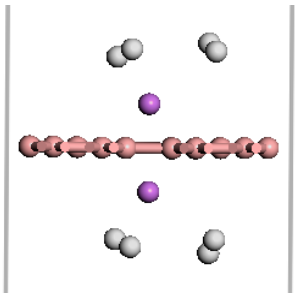

(i)

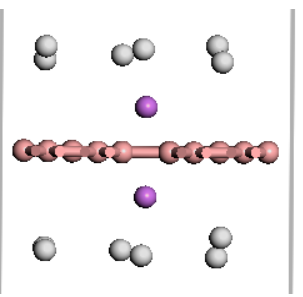

(j)

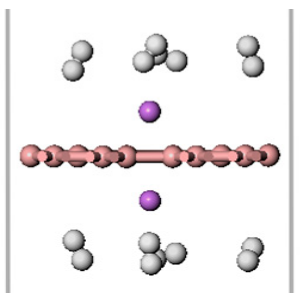

(k)

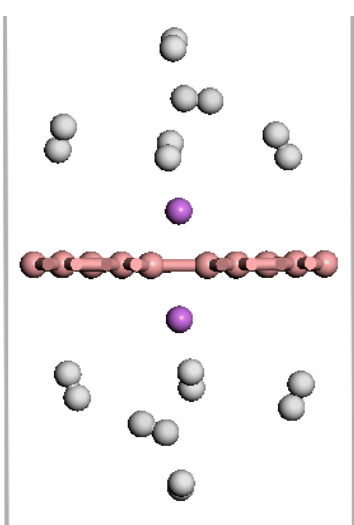

(1)

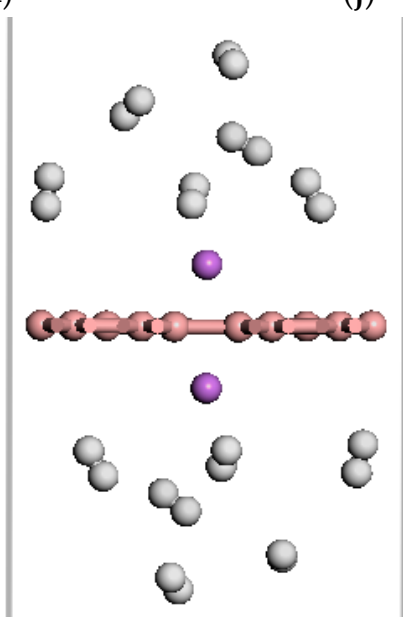

(m)

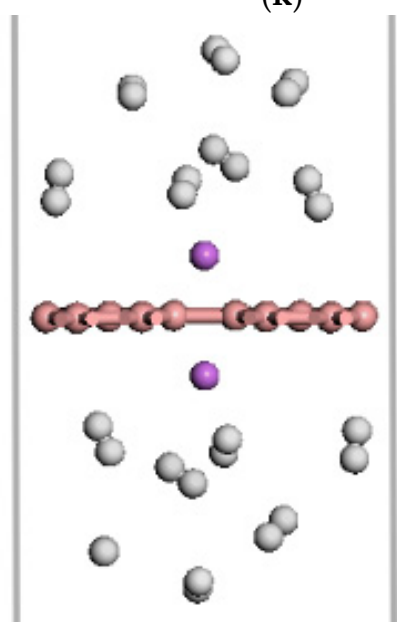

(n)

Figure 5. The optimized atomic structures of the $\mathrm{Li}-\beta_{12}$-borophene/ $\mathrm{H}_{2}$. (a-g) are $1 \sim 7 \mathrm{H}_{2}$ molecules adsorption on $\mathrm{Li}-\beta_{12}$-borophene system. (h-n) are $2 \sim 14 \mathrm{H}_{2}$ molecules adsorption on $2 \mathrm{Li}-\beta_{12}$-borophene system. The pink, purple and white balls in this and aforementioned figures express $\mathrm{B}, \mathrm{Li}$ and $\mathrm{H}$ atoms, respectively. 
Table 1. The adsorption energy, average adsorption energy, the distance between $\mathrm{H}$ and $\mathrm{H}\left(\mathrm{r}_{\mathrm{H}-\mathrm{H}}\right)$, the distance between $\mathrm{H}$ and $\mathrm{Li}$ of $\mathrm{Li}-\beta_{12}$-borophene system $\left(\mathrm{r}_{\mathrm{H}-\mathrm{Li}}\right)$.

\begin{tabular}{ccccccccc}
\hline & $\mathbf{N u m b e r}$ of $\mathbf{H}_{\mathbf{2}}$ & $\mathbf{1} \mathbf{H}_{\mathbf{2}}$ & $\mathbf{2} \mathbf{H}_{\mathbf{2}}$ & $\mathbf{3} \mathbf{H}_{\mathbf{2}}$ & $\mathbf{4} \mathbf{H}_{\mathbf{2}}$ & $\mathbf{5} \mathbf{H}_{\mathbf{2}}$ & $\mathbf{6} \mathbf{H}_{\mathbf{2}}$ & $\mathbf{7} \mathbf{H}_{\mathbf{2}}$ \\
\cline { 2 - 8 } & $E_{\text {ads }} / \mathrm{eV}$ & -0.247 & -0.281 & -0.154 & -0.179 & -0.139 & -0.169 & -0.134 \\
\multirow{2}{*}{$\mathrm{Li}-\beta_{12}$-borophene } & $E_{\text {ads }}(\mathrm{DFT}-\mathrm{D}) / \mathrm{eV}$ & -0.385 & -0.388 & -0.251 & -0.147 & -0.160 & -0.167 & -0.142 \\
& $\bar{E}_{\text {ads }} / \mathrm{eV} / \mathrm{H}_{2}$ & -0.247 & -0.213 & -0.194 & -0.190 & -0.181 & -0.178 & -0.173 \\
& $\bar{E}_{\text {ads }}(\mathrm{DFT}-\mathrm{D}) / \mathrm{eV} / \mathrm{H}_{2}$ & -0.385 & -0.387 & -0.286 & -0.251 & -0.233 & -0.222 & -0.210 \\
& $\mathrm{r}_{\mathrm{H}-\mathrm{H} / \AA}$ & 0.756 & 0.757 & 0.753 & 0.755 & 0.753 & 0.753 & 0.753 \\
& $\mathrm{r}_{\mathrm{H}-\mathrm{Li} /} \AA$ & 2.164 & 2.169 & 3.813 & 3.810 & 4.661 & 5.667 & 6.368 \\
\hline \multirow{2}{*}{$2 \mathrm{Li}-\beta_{12}$-borophene } & $\mathbf{N u m b e r}$ of $\mathbf{H}_{\mathbf{2}}$ & $\mathbf{2} \mathbf{H}_{\mathbf{2}}$ & $\mathbf{4} \mathbf{H}_{\mathbf{2}}$ & $\mathbf{6} \mathbf{H}_{\mathbf{2}}$ & $\mathbf{8} \mathbf{H}_{\mathbf{2}}$ & $\mathbf{1 0 ~ \mathbf { H } _ { \mathbf { 2 } }}$ & $\mathbf{1 2} \mathbf{H}_{\mathbf{2}}$ & $\mathbf{1 4} \mathbf{H}_{\mathbf{2}}$ \\
\hline & $\bar{E}_{\text {ads }}(\mathrm{DFT}-\mathrm{D}) / \mathrm{eV} / \mathrm{H}_{2}$ & -0.381 & -0.298 & -0.274 & -0.262 & -0.230 & -0.226 & -0.220 \\
\hline
\end{tabular}

\subsubsection{Electronic Properties of $\mathrm{Li}-\beta_{12}-$ Borophene $/ \mathrm{H}_{2}$}

The density of states (DOS) reflects the number of states of the unit energy, which is important in further understanding the interaction between $\mathrm{H}_{2}$ and $\mathrm{Li}$ - $\beta_{12}$-borophene. The partial density of states (PDOS) of $\mathrm{Li}-\beta_{12}$-borophene $/ \mathrm{H}_{2}$ is shown in Figure 6. Obvious hybridizations between the Li-s orbit and $\mathrm{H}$-s orbit can be found in $2.0 \mathrm{eV} \sim 5.0 \mathrm{eV}$, which demonstrates a strong interaction between $\mathrm{H}_{2}$ and $\mathrm{Li}$ atoms. With an increase in the number of $\mathrm{H}_{2}$ molecules, the peak values of $\mathrm{H}_{2}$ molecules become smaller and further away from the fermi level. This indicates that the interaction between $\mathrm{H}_{2}$ molecules and $\mathrm{Li}-\beta_{12}$-borophene weakens, which is consistent with the average adsorption energy becoming smaller. Another overlap between the B-p and $\mathrm{H}-\mathrm{s}$ orbits was found at $-10.0 \mathrm{eV} 5.0 \mathrm{eV}$. Upon the adsorption of the second $\mathrm{H}_{2}$ molecule, the $\mathrm{H}$ 1s orbit peaks move to the lift, implying an increased stability in the system. This is consistent with the increase in the Eads value after the second $\mathrm{H}_{2}$ addition. With an increase in the number of $\mathrm{H}_{2}$ molecules (an expected in the second $\mathrm{H}_{2}$ ), the $\mathrm{H}-\mathrm{s}$ orbits move to the right and the peak values become smaller, which indicates that the interaction between $\mathrm{H}_{2}$ and $\beta_{12}$-borophene becomes increasingly weaker. This conclusion is consistent with the decrease in the average adsorption energy $\left(\bar{E}_{a d s}\right)$. The B-p and Li-s orbits also have hybridization, which implies an interaction between the $\mathrm{Li}$ and $\mathrm{B}$ atoms. The peaks near and below the fermi surface are mostly contributed by the B-s orbits, which means that the $\mathrm{H}_{2}$ molecules and Li atom have less influence on the $\beta_{12}$-borophene. The comparison of the PDOS of a single $\mathrm{Li}$ - $\beta_{12}$-borophene show that the interaction between $\mathrm{Li}$ atom and $\beta_{12}$-borophene is weakened due to the adsorption of $\mathrm{H}_{2}$ molecules. The PDOS of two Li- $\beta_{12}$-borophene $/ \mathrm{H}_{2}$ consistent with this analysis.

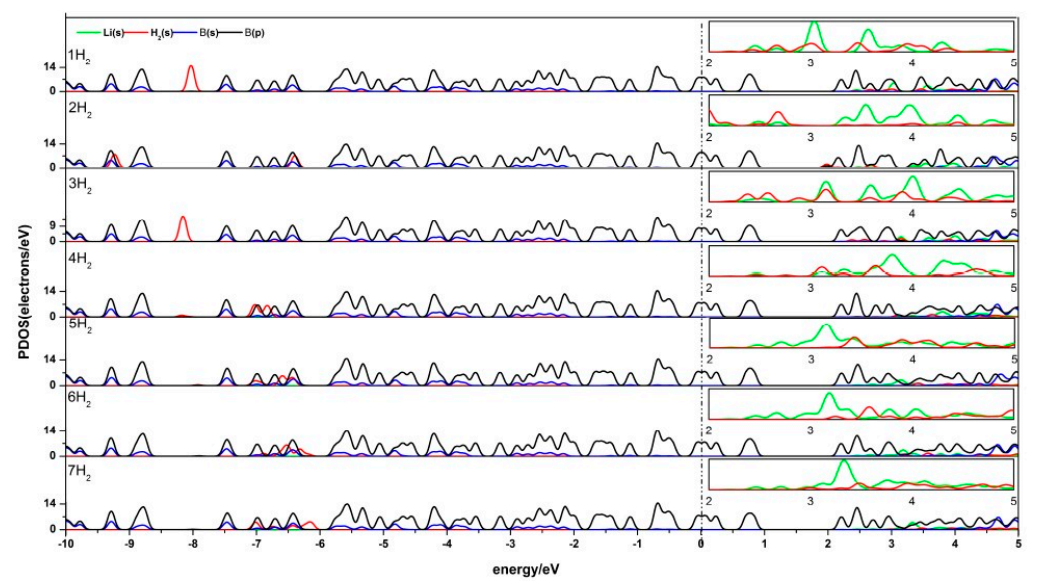

Figure 6. PDOS of Li- $\beta_{12}$-borophene with $1-7 \mathrm{H}_{2}$ molecules adsorbing. (The PDOS of Li-s orbit and H-s orbit in the range of $2.0 \mathrm{eV} \sim 5.0 \mathrm{eV}$ is enlarged as shown in the small box above each corresponding figure.) 
The bonding strength between atoms can be quantitatively analyzed based on the Mulliken charge population and bond population. Table 2 shows the Mulliken charge population before and after one $\mathrm{H}_{2}$ moleculebecomes absorbed on the $\mathrm{Li}-\beta_{12}$-borophene. $\mathrm{H}(1)$ and $\mathrm{H}$ (2) represent the two $\mathrm{H}$ atoms of the adsorbed $\mathrm{H}_{2}$ molecule; while $\mathrm{B} 1, \mathrm{~B} 5$ and $\mathrm{B} 6$ are three $\mathrm{B}$ atoms that transfer the greatest amount of charge in the $\beta_{12}$-borophene (as shown in Figure 1). The two $\mathrm{H}$ atoms have charges of $0.06 \mathrm{e}$ and $0.05 \mathrm{e}$, respectively. In contrast, the $\mathrm{Li}$ atom loses $1.40 \mathrm{e}$, which occurs mainly in the $\mathrm{H}$ and $\mathrm{Li}$ atomic orbits. The Li atom transfers charge to the $\mathrm{H}_{2}$ molecules, resulting in the $\mathrm{H}_{2}$ molecules carrying more negative charge and $\mathrm{Li}$ atom showing positive charge. The interaction between the $\mathrm{H}_{2}$ molecules and the $\mathrm{Li}$ atom is consistent with the conclusion of the PDOS analysis. In addition, the B atoms obtains charge, with this charge transfer mainly occurring in the B-2p orbits and H-s orbits. This is in contrast with the Mulliken charge population of the $\beta_{12}$-borophene $/ \mathrm{H}_{2}$, in which the charge transfer mainly occurs in $\mathrm{H}$ and $\mathrm{B}$ atoms forming a covalent bond of $\mathrm{H}-\mathrm{B}$ that is not favorable for the desorption of $\mathrm{H}_{2}$. Due to the $\beta_{12}$-borophene being modified by $\mathrm{Li}$ atoms, $\mathrm{H}_{2}$ molecules and $\mathrm{B}$ atoms only have small interactions, resulting in the $\mathrm{H}_{2}$ molecules physically adsorbing on the Li- $\beta_{12}$-borophene. This is conducive for $\mathrm{H}_{2}$ desorption and increases the hydrogen storage capacity.

Table 2. Mulliken population analysis of the $\mathrm{Li}-\beta_{12}$-borophene before and after one $\mathrm{H}_{2}$ molecule adsorption.

\begin{tabular}{ccccccc}
\hline & \multicolumn{6}{c}{ Mulliken } \\
\cline { 2 - 7 } Atom & \multicolumn{3}{c}{ Before Adsorption/e } & \multicolumn{2}{c}{ After Adsorption/e } \\
\cline { 2 - 7 } & $\mathbf{s}$ & $\mathbf{p}$ & Charge & $\mathbf{s}$ & $\mathbf{p}$ & Charge \\
\hline H (1) & 1.0 & & & 1.06 & & -0.06 \\
H (2) & 1.0 & & & 1.05 & & -0.05 \\
B1 & 0.82 & 2.18 & 0 & 0.83 & 2.36 & -0.19 \\
B5 & 0.74 & 2.23 & 0.03 & 0.75 & 2.40 & -0.15 \\
B6 & 0.65 & 2.40 & -0.05 & 0.65 & 2.40 & -0.05 \\
Li & 3 & 0 & 0 & 1.60 & & 1.40 \\
\hline
\end{tabular}

\section{Conclusions}

In summary, we performed a study on hydrogen storage properties of pure $\beta_{12}$-borophene and Li-decorated $\beta_{12}$-borophene through DFT calculations. It is found that $\mathrm{H}_{2}$ molecules are mainly adsorbed on pure $\beta_{12}$-borophene as chemical adsorption with an adsorption energy of $-0.536 \mathrm{eV}$. The $\mathrm{H}_{2}$ molecules are dissociated into two $\mathrm{H}$ atoms, which tend to the bridge of two $\mathrm{B}$ site and the $\mathrm{H}-\mathrm{B}$ bond to form covalent bond. In order to improve the hydrogen storage performance of pure $\beta_{12}$-borophene and increase the hydrogen storage capacity, we use the Li atom to modify the $\beta_{12}$-borophene. It is found that a single $\mathrm{Li}$ atom adsorbed on the center of Boron ring with the adsorption energy $-3.088 \mathrm{eV}$, the $\mathrm{Li}-\beta_{12}$-borophene can adsorb up to $7 \mathrm{H}_{2}$ molecules with the average adsorption energy of $-0.210 \mathrm{eV} / \mathrm{H}_{2}$. The charge transfer of the $\mathrm{Li}-\beta_{12}$-borophene $/ \mathrm{H}_{2}$ is that $\mathrm{H}$ and $\mathrm{B}$ atoms lose electron, $\mathrm{Li}$ atom get electron. We use two Li atoms to modify $\beta_{12}$-borophene to increase its hydrogen storage capacity. It is find that the two Li atoms are located at the same position on both sides of the same boron hole. $2 \mathrm{Li}-\beta_{12}$-borophene system can adsorb up to $14 \mathrm{H}_{2}$ molecules and the hydrogen storage capacity up to $10.85 \mathrm{wt} \%$. The average adsorption energy is range of -0.381 to $-0.220 \mathrm{eV} / \mathrm{H}_{2}$, which is necessary for practical application $[3,4]$.

Acknowledgments: This work was supported by the National Natural Science Foundation of China (grant number 51562022), the fund for the State Key Laboratory of Advanced Processing and Recycling of Non-Ferrous Metals, Lanzhou University of Technology (grant number SKLAB02014004), the Basic Scientific Research Foundation for Gansu Universities of China (grant number 05-0342), the Science and Technology Project of Lanzhou City (grant number 2011-1-10), the Natural Science Foundation of Gansu Province (Grant No. 17JR5RA123), and the Special Program for Applied Research on Super Computation of the NSFC-Guangdong Joint Fund (second phase). 
Author Contributions: Yuhong Chen designed the project, Tingting Liu performed the calculations, Yuhong Chen and Tingting Liu prepared the manuscript, Haifeng Wang and Cairong Zhang revised the paper, Meiling Zhang and Lihua Yuan analyzed the data, and all authors discussed the results and commented on the manuscript.

Conflicts of Interest: The authors declare no conflict of interest.

\section{References}

1. Song, Y.; Guo, Z.X.; Yang, R. Influence of selected alloying elements on the stability of magnesium dihydride storage applications: A first-principles investigation. Phys. Rev. B 2004, 69, 094205. [CrossRef]

2. Schlapbach, L.; Züttel, A. Hydrogen-storage materials for mobile applications. Nature 2001, 414, $353-358$. [CrossRef] [PubMed]

3. Rosi, N.L.; Eckert, J.; Eddaoudi, M.; Vodak, D.T.; Kim, J.; O’Keeffe, M.; Yaghi, O.M. Hydrogen storage in microporous metal-organic frameworks. Science 2003, 300, 1127-1129. [CrossRef] [PubMed]

4. Han, S.S.; Goddard, W.A. Lighium-doped metal-organic frameworks for reversible $\mathrm{H}_{2}$ storage at ambient temperature. J. Am. Chem. Soc. 2007, 129, 8422-8423. [CrossRef] [PubMed]

5. U.S. Department of Energy. Hydrogen, Fuel Cells Program: FY Annual Progress Report; U.S. Department of Energy: Washington, DC, USA, 2014.

6. Seenithurai, S.; Pandyan, R.K.; Kumar, S.V.; Saranya, C.; Mahendran, M. Al-decorated carbon nanotube as the molecular hydrogen storage medium. Int. J. Hydrog. Energy 2014, 39, 11990-11998. [CrossRef]

7. Wu, M.H.; Gao, Y.; Zhang, Z.Y.; Zeng, X.C. Edge-decorated graphene nanoribbons by scandium as hydrogen storage media. Nanoscale 2012, 4, 915-920. [CrossRef] [PubMed]

8. Mannix, A.J.; Zhou, X.F.; Kiraly, B.; Wood, J.D.; Alducin, D.; Myers, B.D.; Liu, X.; Fisher, B.L.; Santiago, U.; Guest, J.R.; et al. Synthesis of borophenes: Anisotropic, Two-dimensional boron polymorphs. Science 2015, 350, 1513-1516. [CrossRef] [PubMed]

9. Zhang, Z.H.; Yang, Y.; Gao, G.Y.; Yakobson, B.I. Two-Dimensional Boron Monolayers Mediated by metal Substrates. Angew. Chem. Int. Ed. Engl. 2015, 127, 13022-13026. [CrossRef] [PubMed]

10. Feng, B.J.; Zhang, J.; Zhong, Q.; Li, W.B.; Li, S.; Li, H.; Cheng, P.; Meng, S.; Chen, L.; Wu, K.H. Experimental realization of two-dimensional boron sheets. Nat. Chem. 2016, 8, 563. [CrossRef] [PubMed]

11. Feng, B.J.; Zhang, J.; Liu, R.Y.; Iimori, T.; Lian, C.; Li, H.; Chen, L.; Wu, K.H.; Meng, S.; Komori, F.; et al. Direct evidence of metallic bands in a monolayer boron sheet. Phys. Rev. B 2016, 94, 041408. [CrossRef]

12. Padilha, J.E.; Miwa, R.H.; Fazzio, A. Directional dependence of the electronic and transport properties of 2D borophene and borophane. Phys. Chem. Chem. Phys. 2016, 18, 25491-25496. [CrossRef] [PubMed]

13. Mortazavi, B.; Rahaman, O.; Dianat, A.; Rabczuk, T. Mechanical responses of borophene sheets: A first-principles study. Phys. Chem. Chem. Phys. 2016, 18, 27405. [CrossRef] [PubMed]

14. Wang, H.F.; Li, Q.F.; Gao, Y.; Miao, F.; Zhou, X.F.; Wan, X.G. Strain effects on borophene: Ideal strength, negative Possion's ration and phonon instability. New J. Phys. 2016, 18, 073016. [CrossRef]

15. Liu, Y.; Dong, Y.J.; Tang, Z.; Wang, X.F.; Wang, L.; Hou, T.G.; Lin, H.P.; Li, Y.Y. Stable and metallic borophene nanoribbons from first-principles calculations. J. Mater. Chem. C 2016, 4, 6380-6385. [CrossRef]

16. Novoselov, K.S.; Geim, A.K.; Morozov, S.V.; Jiang, D.; Zhang, Y.; Dubonos, S.V.; Grigorieva, I.V.; Firsov, A.A. Electric field effect in atomically thin carbon films. Science 2004, 306, 666-669. [CrossRef] [PubMed]

17. Chen, X.F.; Wang, L.; Zhang, W.T.; Zhang, J.L.; Yuan, Y.Q. Ca-decorated borophene as potential candidates for hydrogen storage: A first-principle study. Int. J. Hydrog. Energy 2017, 42, 20036-20045. [CrossRef]

18. Wang, Y.H.; Meng, Z.S.; Liu, Y.Z.; You, D.; Wu, K.; Lv, J.; Wang, X.Z.; Deng, K.M.; Rao, D.; Lu, R.F. Lithium decoration of three dimensional boron-doped graphene frameworks for high-capacity storage. Appl. Phys. Lett. 2015, 106, 2721. [CrossRef]

19. Reunchan, P.; Jhi, S.H. Metal-dispersed porous graphene for hydrogen storage. Appl. Phys. Lett. 2011, $98,93103$. [CrossRef]

20. Ao, Z.M.; Jiang, Q.; Zhang, R.Q.; Tan, T.T.; Li, S. Al doped graphene: A promising material for hydrogen storage at room temperature. J. Appl. Phys. 2009, 105, 074307. [CrossRef]

21. Faye, O.; Eduok, U.; Szpunar, J.; Szpunar, B.; Samoura, A.; Beye, A. Hydrogen Storage on bare Cu atom and Cu-functionalized boron-doped graphene: A first principles study. Int. J. Hydrog. Energy 2017, 42, 4233-4243. [CrossRef] 
22. Faye, O.; Szpunar, J.A.; Szpunar, B.; Beye, A.C. Hydrogen adsorption and storage on Palladium-functionalized graphene with NH-dopant: A first principles calculation. Appl. Surf. Sci. 2017, 392, 362-374. [CrossRef]

23. Yuan, L.H.; Chen, Y.H.; Kang, L.; Zhang, C.R.; Wang, D.B.; Wang, C.N.; Zhang, M.L.; Wu, X.J. First-principles investigation of hydrogen storage capacity of Y-decorated porous graphene. Appl. Surf. Sci. 2017, 399, 463-468. [CrossRef]

24. Zhao, Y.; Kim, Y.H.; Dillion, A.C.; Heben, M.J.; Zhang, S.B. Hydrogen storage in novel organometallic buckyballs. Phys. Rev. Lett. 2005, 94, 155504. [CrossRef] [PubMed]

25. Yoon, M.; Yang, S.Y.; Hicke, C.; Wang, E.; Geohegan, D.; Zhang, Z.Y. Calcium as the superior coating metal in functionalization of carbon fullerenes for high-capacity hydrogen storage. Phys. Rev. Lett. 2008, 100, 206806. [CrossRef] [PubMed]

26. Guo, J.; Liu, Z.G.; Liu, S.Q.; Zhao, X.H.; Huang, K.L. High-capacity hydrogen storage medium: Ti doped fullerene. Appl. Phys. Lett. 2011, 98, 023107. [CrossRef]

27. Clark, S.J.; Segall, M.D.; Pickard, C.J.; Hasnip, P.J.; Probert, M.; Refson, K.R.; Payne, M.C. First Principles methods using CASTEP. Z. Kristallogr. 2005, 220, 567-570. [CrossRef]

28. Perdew, J.P.; Burke, K.; Ernzerhof, M. Generalized Gradient Approximation Made Simple. Phys. Rev. Lett. 1996, 77, 3865-3868. [CrossRef] [PubMed]

29. Bjorkman, T.; Gulans, A.; Krasheninnikov, A.V.; Nieminen, R.M. Van der Waals Bonding in Layered Compounds from Advanced Density-Functional First-Principles Calculations. Phys. Rev. Lett. 2012, 108, 235502. [CrossRef] [PubMed]

30. Vanderbilt, D. Soft self-consistent pseudopotentials in generalized eigenvalue formalism. Phys. Rev. B 1990, 41, 7892-7895. [CrossRef]

31. Hu, W.; Xia, N.; Wu, X.; Li, Z.; Yang, J. Silicene as a highly sensitive molecule sensor for $\mathrm{NH}_{3}, \mathrm{NO}$ and $\mathrm{NO}_{2}$. Phys. Chem. Chem. Phys. 2014, 16, 6957-6962. [CrossRef] [PubMed]

32. Chen, Y.H.; Wang, J.; Yuan, L.H.; Zhang, M.L.; Zhang, C.R. Sc-Decorated Porous Graphene for High-Capacity Hydrogen Storage: First-Principles Calculations. Materials 2017, 10, 894. [CrossRef] [PubMed]

33. Wu, X.; Dai, J.; Zhuo, Z.; Yang, J.; Zeng, X. Two-Dimensional Boron Monolayer Sheets. ACS Nano 2012, 6 , 7443-7453. [CrossRef] [PubMed]

34. Peng, B.; Zhang, H.; Shao, H.; Ning, Z.; Xu, Y.; Ni, G.; Lu, H.; Zhang, D.; Zhu, H. Stability and strength of atomically thin borophene from first principles calculations. Mater. Res. Lett. 2017, 5, 399-407. [CrossRef]

35. Peng, B.; Zhang, H.; Shao, H.Z.; Xu, Y.F.; Zhang, R.J.; Zhua, H.Y. Electronic, Optical, and thermodynamic properties of borophene from first-principle calculations. J. Mater. Chem. C 2016, 4, 3592-3598. [CrossRef]

36. Pan, C.C.; Chen, Y.H.; Wu, N.; Zhang, M.L.; Yuan, L.H.; Zhang, C.R. A First Principles Study of $\mathrm{H}_{2}$ Adsorption on $\mathrm{LaNiO}_{3}(001)$ Surfaces. Materials 2017, 10, 36. [CrossRef] [PubMed]

37. Ataca, C.; Akturk, E.; Ciraci, S.; Ustunel, H. High-capacity hydrogen storage by metallized graphene. Appl. Phys. Lett. 2008, 93. [CrossRef]

38. Sun, Q.; Wang, Q.; Jena, P.; Kawazoe, Y. Clustering of Ti on a C60 surface and its effect on hydrogen storage. J. Am. Chem. Soc. 2005, 127, 14582-14583. [CrossRef] [PubMed]

39. Doll, K.; Harrison, N.M.; Saunders, V.R. A density functional study of lithium bulk and surfaces. J. Phys.-Condens. Matter 1999, 11, 5007-5019. [CrossRef]

40. Mulliken, R.S. Molecular Compounds and Their Spectra. V. Orientation in Molecular Complexes. J. Chem. Phys. 1955, 23, 1833-1840. [CrossRef]

41. An, H.; Liu, C.S.; Zeng, Z.; Fan, C.; Ju, X. Li-doped B2C graphene as potential hydrogen storage medium. Appl. Phys. Lett. 2011, 98, 173101-173103. [CrossRef]

(C) 2017 by the authors. Licensee MDPI, Basel, Switzerland. This article is an open access article distributed under the terms and conditions of the Creative Commons Attribution (CC BY) license (http://creativecommons.org/licenses/by/4.0/). 